\title{
Single Event Effects Testing of the Linfinity SG1525A Pulse Width Modulator Controller
}

\author{
J. W. Howard Jr. ${ }^{1}$, M. A. Carts ${ }^{2}$, K. A. LaBel ${ }^{3}$, J. D. Forney ${ }^{1}$ and T. L. Irwin ${ }^{4}$ \\ 1. Jackson and Tull Chartered Engineers, Washington DC 20018 \\ 2. Raytheon ITSS, Lanham, MD 20706 \\ 3. NASA Goddard Space Flight Center, Greenbelt, MD 20771 \\ 4. QSS Group, Inc., Seabrook, MD 20706
}

\begin{abstract}
The Linfinity SG1525A Pulse Width Modulator Controller was investigated for Single Event Effects. While no latchup events were observed, a temporal overlap of the outputs, that could be destructive, was observed for all LETs used.
\end{abstract}

\section{Introduction}

Pulse Width Modulator (PWM) Controllers are the heart of switching power supply systems in development today. The PWMs considered here have the same integration advantages as many other controllers but it also includes the interface drivers for the follow-on power Field Effect Transistors (FET). Previous work on these types of devices looked into the required test methodologies [1] and the impact of radiation on the soft start and shutdown circuits of typically incorporated in the technology [2]. Taking advantage of this previous work this study was undertaken to determine the single event destructive and transient susceptibility of the Linfinity SG1525A Pulse Width Modulator Controller. The device was monitored for transient interruptions in the output signals and for destructive events induced by exposing it to a heavy ion beam at the Texas A\&M University Cyclotron Single Event Effects Test Facility. After exposing these devices to the beam, a new upset mode has been identified that can lead to catastrophic power supply system failure if this event would occur while drive power FETs off the two device outputs. The devices and the test methods used will be described first. This will be followed by a brief description of the data collected to date (not all data can be presented with the length constraints of the summary) and a summary of the key results.

\section{Devices and Test Methods}

The SG1525A Pulse Width Modulator controller integrated circuit contains all logic and drivers required to implement all types of switching power supplies. It contains a $1 \%$ voltage reference, an oscillator with synchronization capability (to synchronize multiple devices), a pair of power FET drivers, programmable dead time control (to ensure one FET is off before the other begins to turn on), soft start circuitry, shutdown control, under-voltage shutdown, and a pulse latch-off circuit (to prevent any pulse, once terminated, from turning on again). The oscillator runs from 100 to $500 \mathrm{kHz}$. The device operates from 8 to $35 \mathrm{~V}_{\mathrm{dc}}$ input, and the logic and the output drivers have independent supplies $\left(V_{\text {in }}\right.$ and $V_{c}$, respectively).

The device under test (DUT) was packaged in a 16-pin ceramic DIP. The DUTs tested with heavy ions were de-lidded to allow the limited range ions access to the active layer of the die with the cleave point just below the upper layer of the package, leaving the lower and middle layers with leads intact.

The application for which these tests were conducted has two DUTs, termed the Master and Slave devices. They are synchronized together via the SYNC line out of the Master device and the Master device driven by an external $200 \mathrm{kHz}$ reference frequency source (Normal mode). In the absence of the external frequency reference, the application circuit was designed to self-oscillate at some lower frequency, thus not losing total functionality (Free-run mode). The source voltage for both the DUT logic and the output driver collectors was 10.75 Volts $\left(V_{d c}\right)$. Each DUT in the application circuit drives a pair of FETs, each at half the oscillator frequency, and each out of phase with the other. The FETs drive either end of 
a center-tap grounded transformer primary to ground when driven high (turned $\mathrm{ON}$ ) by the DUT. The output of each DUT's transformer had multiple outputs, which were rectified and filtered.

The DUT is designed for voltage feedback in order to modulate the width of each output pulse to maintain final filtered voltage stability. However, this application drives the outputs at maximum $(50 \%$ duty cycle minus dead time of approximately $5 \%$ ) with no feedback.

Figure lshows the functional block diagram of the SG1525A. The output of the DUT is a pair of pulse trains for driving FETs, which drive a transformer, whose output(s) are then rectified and filtered. Reproducing the totality of this circuit, especially the FETs and transformer, was deemed cumbersome and of little value. Instead of trying to determine SEE from the filtered DC of the circuit's output, the DUT outputs were monitored, with simulated FET loading, for deviation from the nominal waveforms. Knowledge of the dynamics of the application circuit will allow the application circuit designers to determine the end effects of any observed single events on the output voltages.

The application circuit's topology was reproduced for the DUTs, with some limitations and modifications. The input voltage source was driven by an HP6626A power supply instead of a switching supply and the reference frequency input was supplied by a pulse generator. Also, the gates of the power FETs (and all following circuitry, transformers, rectifiers, filter capacitors) were simulated by an equivalent capacitance. The application FETs, $100 \mathrm{~V} \mathrm{~N}$ channel enhancement mode radiation hardened FETs, have a $\mathrm{C}_{\text {iss }}$ (gatesource capacitance) of about $1000 \mathrm{pF}$, typically, and $\mathrm{C}_{\mathrm{rss}}$ (gate-drain capacitance) of $45 \mathrm{pf}$. The Miller effect can magnify the effect of $\mathrm{C}_{\mathrm{rss}}$, but at a $V_{d s}$ of only 10.75 Volts this did not have a significant effect in this case. The FET was simulated with a mica, $1100 \mathrm{pF}$ capacitance to ground. The application circuit's $100 \Omega$ series gate resistors were included in the test circuit.

Two iterations of the two DUT circuit were built using good RF practices (specifically, ground plane and layout which minimized high frequency and power supply bypass capacitor trace lengths). Power and frequency reference were switched between circuits by relays. DUT outputs were not switched in order to maintain signal edge fidelity. Rather, all eight (two per DUT) probe coaxial cables were brought out, so that switching operation from one DUT to another could be affected fairly rapidly. Relays were actuated by otherwise unused power supply outputs. Each DUT within a circuit was supplied by a separate supply so that individual DUT currents could be monitored (See Figure 2).

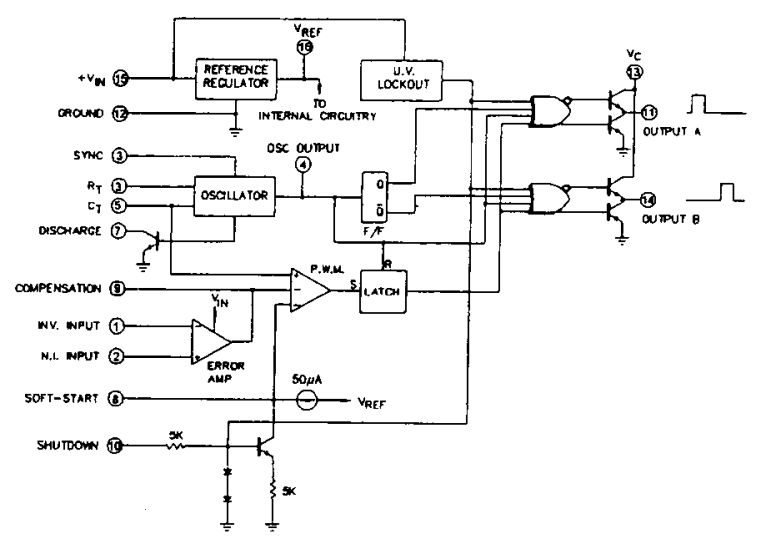

Figure 1. Functional Block diagram of the SG1525A.

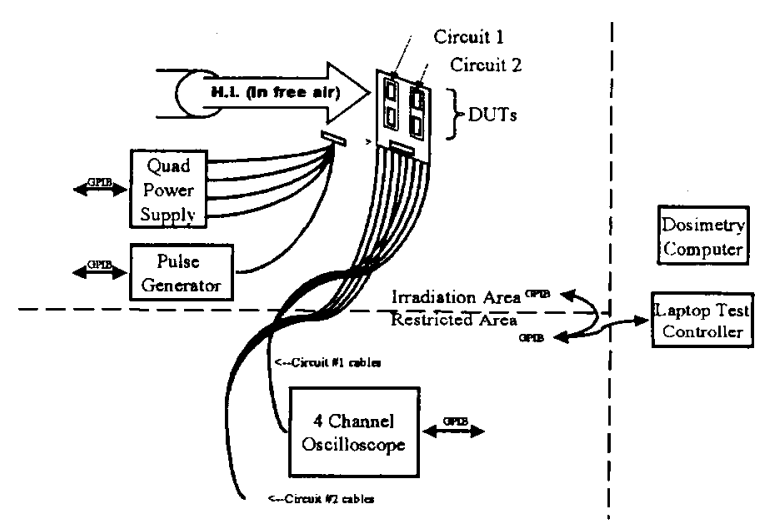

Figure 2. Block diagram of the test setup

The DUT outputs were monitored directly at the DUT IC pins. Normally, low capacitance FET probes would be used to monitor signals with low loading effect, but in this case the $8 \mathrm{~V}$ linear range of the probes was too low to monitor the almost $11 \mathrm{~V}_{\mathrm{pp}}$ signals. Instead, a high impedance high bandwidth probing technique was employed. A 5 $\mathrm{k} \Omega$ resistor and a $50 \Omega$ resistor (involving a length of $50 \Omega$ coax plus the scope's $50 \Omega$ termination resistance on the far end) formed a resistive divider (giving input signals at the scope reduced 
by approximately a factor of 100 ). The $5.05 \mathrm{k} \Omega$ load on the DUT pin was negligible. Also note that the output $B$ signal was offset in voltage at the scope by approximately $10 \mathrm{mV}$, allowing for better monitoring of both output channels.

The triggering mode used to capture radiation-induced events was set to the pulse mode. In this mode, the scope will trigger when it sees an input pulse that is smaller than a given amount of time. This method was chosen since the expected error types would either be the shortening of one output pulse or the loss of a sequence of output pulses.

The test flow for these devices included testing of both modes of operation (Normal and Free-run) and exposing both the Master and Slave devices to the ion beam. For these four test conditions, the output of either the Master or Slave device is monitored at the oscilloscope, leading to eight test conditions at each effective LET. DUT angles of either $0,30,45$, or 60 degrees were used to achieve an effective LET range from 8.6 to $61.3 \mathrm{MeV}-\mathrm{cm}^{2} / \mathrm{mg}$. Finally, on one DUT at the higher normal incidence LET of 53.1 , the $\mathrm{V}_{\mathrm{ds}}$ was raised to 12 volts to perform a worst-case latchup test.

\section{Results}

\section{Single Event Latchup}

Four parts, biased at nominal voltage (10.75 volts), were tested with heavy ions with LETs ranging from 8.6 to $61.3 \mathrm{MeV}-\mathrm{cm}^{2} / \mathrm{mg}$. Additionally, one part was exposed to an ion beam with an LET of 53.1 while biased at 12 volts. These conditions were run while the devices were operated in both the Normal and Free-run modes. In no test condition were any high current conditions observed that would indicate any latchup or other destructive mode.

\section{Single Event Upsets/Transients}

Normal outputs from the SG1525A are shown in Figure 3 for the two modes of operation. When exposed to the heavy ion irradiation, three primary modes of altering this output were observed. These were termed simple, double and overlap. A simple event is one in which the only observable difference is that one output pulse is either shortened or missing.

A double event is one where the output of one of the channels goes high consecutively, rather than alternate with the opposite channel. It should be noted that the majority of the double events were seen on channel B output. However, that is simply due to the triggering scheme used. There is no indication in the data sheet that would indicate either channel having a preference for these double events.

The overlap event is one in which the two channels lose their sync and the output "highs" and "lows" overlap. This overlap condition can exist for very short periods of time to complete overlap. The distribution of percent overlap appears to be uniform in time, indicating no preference or mode for initiating this overlap condition. Finally, it should be stated that the observed overlap events do not necessarily give a complete picture of the events and their true rate. This is due to the triggering scheme used for this testing. Some events that were captured saw the initiation of the overlap condition. However, other events were captured that showed the overlap condition existing prior to the trigger point, indicating that the overlap condition was initiated some time earlier but was not seen until a proper trigger event occurred. However, it is not believed that this loss of sync is a long-term event, as the overlap condition (whether overlapped or not or the percent overlap) did not persist from one trigger event to the next (typical event rates were less than one per second). To get a more accurate indication of the overlap event onset and event rate, more testing with logic triggering (trigger when both outputs go "high") is required.

The second issue to deal with in transient/upset characteristics is the cross section. Figure 4 shows the cross section versus effective LET curves for the Normal and Free-run modes of operation while irradiating and monitoring the Master device. The first comment is that the Weibull fit to the Total cross section appears to be independent of mode of operation (as the Weibull curve plotted is the same for the two figures). This curve shows an onset threshold LET of approximately $5 \mathrm{MeV}-\mathrm{cm}^{2} / \mathrm{mg}$ and a saturation cross section of approximately $1 \times 10^{-4} \mathrm{~cm}^{2}$. The curves for the double and overlap events may show some dependence on the mode of operation. They do show a smaller overall saturation cross section and possibly higher threshold LETs. However, to fully evaluate these statements, additional testing with triggering modes to fully

Nuclear \& Space Radiation

July 21-25, 2003 
capture all double and overlap events is needed as well as testing at LETs less than 8.6.
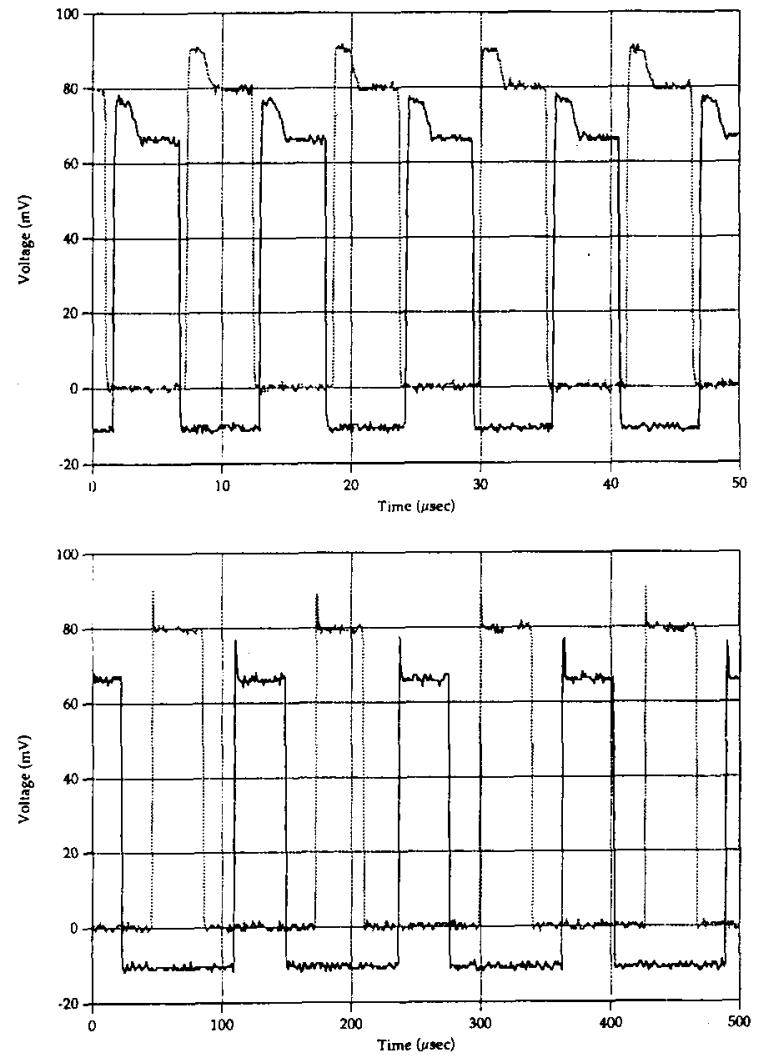

Figure 3. Pre-rad traces for Normal (left) and Free-run (right) modes.

Sample output for each of the modes of upset and the modes of operation are given in Figure 5 and Figure 6. It is easily see that the upper and middle transient conditions for both modes of operation would have little impact on a switching mode power supply system. The bottom transients, on the other hand, could lead to catastrophic conditions and possible power supply burnout.

\section{Summary}

This paper reports on the heavy ion Single Events Effects testing of the Linfinity SG1525A Pulse Width Modulator Controller. The results have shown no catastrophic failure modes. However, a previously unseen event was observed where the outputs of the controller would come into sync, allowing both outputs to be high at the same time. If this event would occur while the controller was used as part of a switching power supply, the path from power to ground would be opened through the two power FETs. This could lead to a catastrophic failure of the power supply. Additional testing is planned to better define the threshold and true event rate and will be included in the final poster and paper.

\section{References}

[1] S.H.Penzin, W.R.Crain, K.B.Crawford, S.J.Hansel and R.Koga, "The SEU in Pulse Width Modulator Controllers with Soft Start and Shutdown Circuits", 1997 IEEE Radiation Effects Data Workshop Record.

[2] S.H.Penzin, W.R.Crain, K.B.Crawford, S.J.Hansel, J.F.Kirshman and R.Koga, "Single Event Effects in Pulse Modulation Controllers", IEEE Transactions on Nuclear Science, Vol. 43, No. 6, December 1996. 

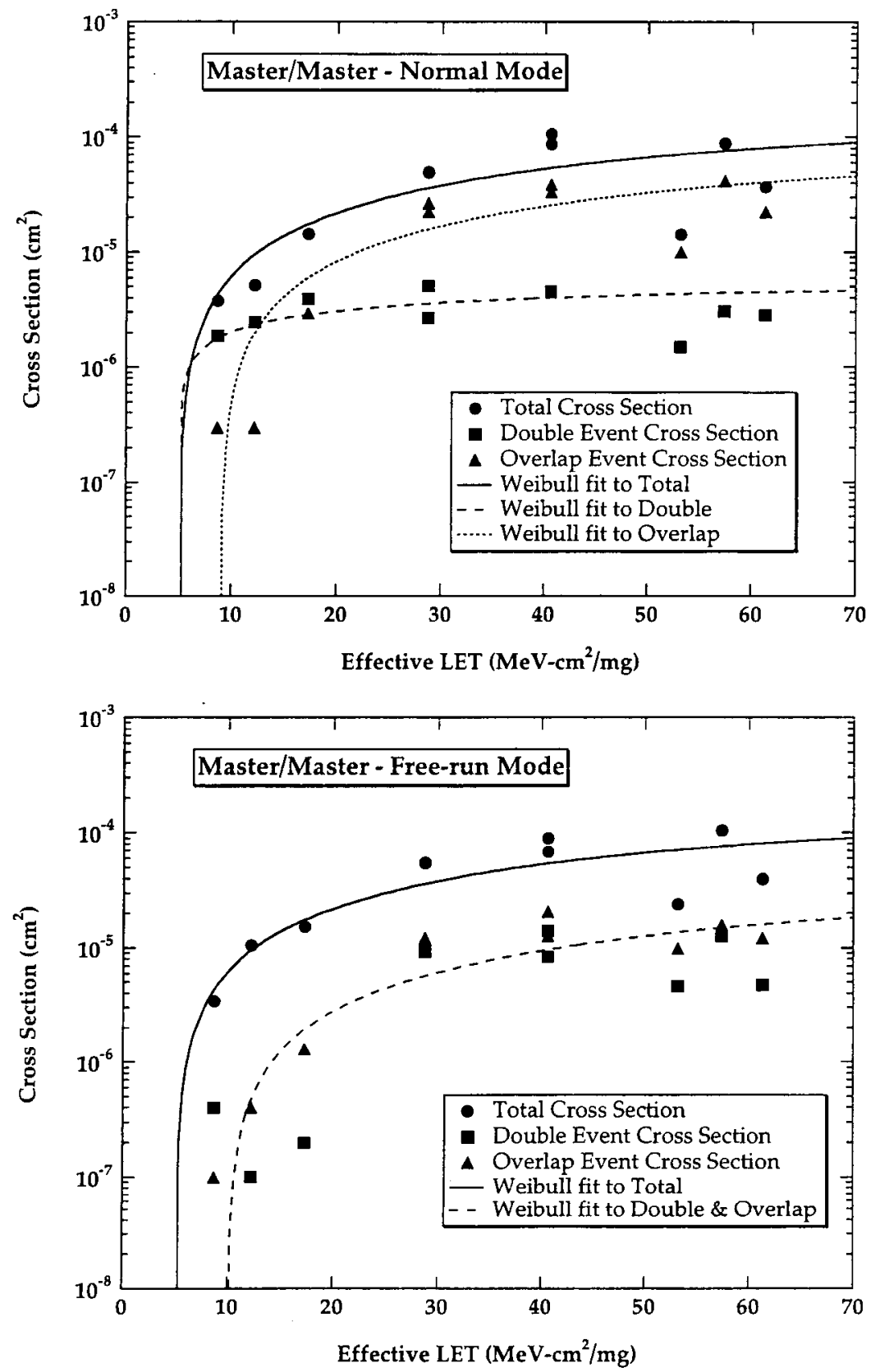

Figure 4. Cross section versus effective LET curves for the Normal mode (left) and Free-run mode (right) of operation, irradiating and observing the Master device. 

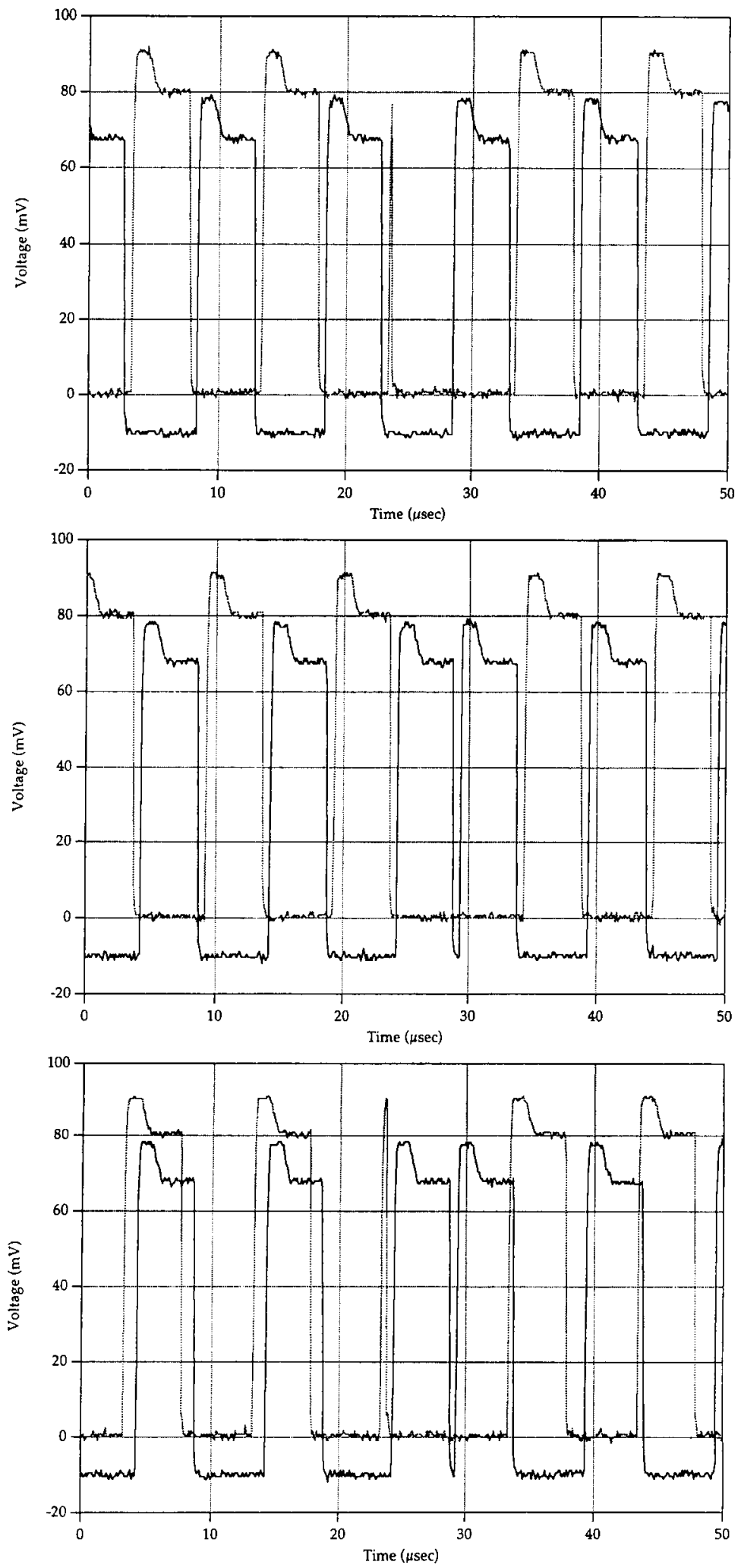

Figure 5. Sample outputs showing simple (top), double (middle), and overlapping (bottom) events. Operation was in Normal mode and the LET was 8.6. 

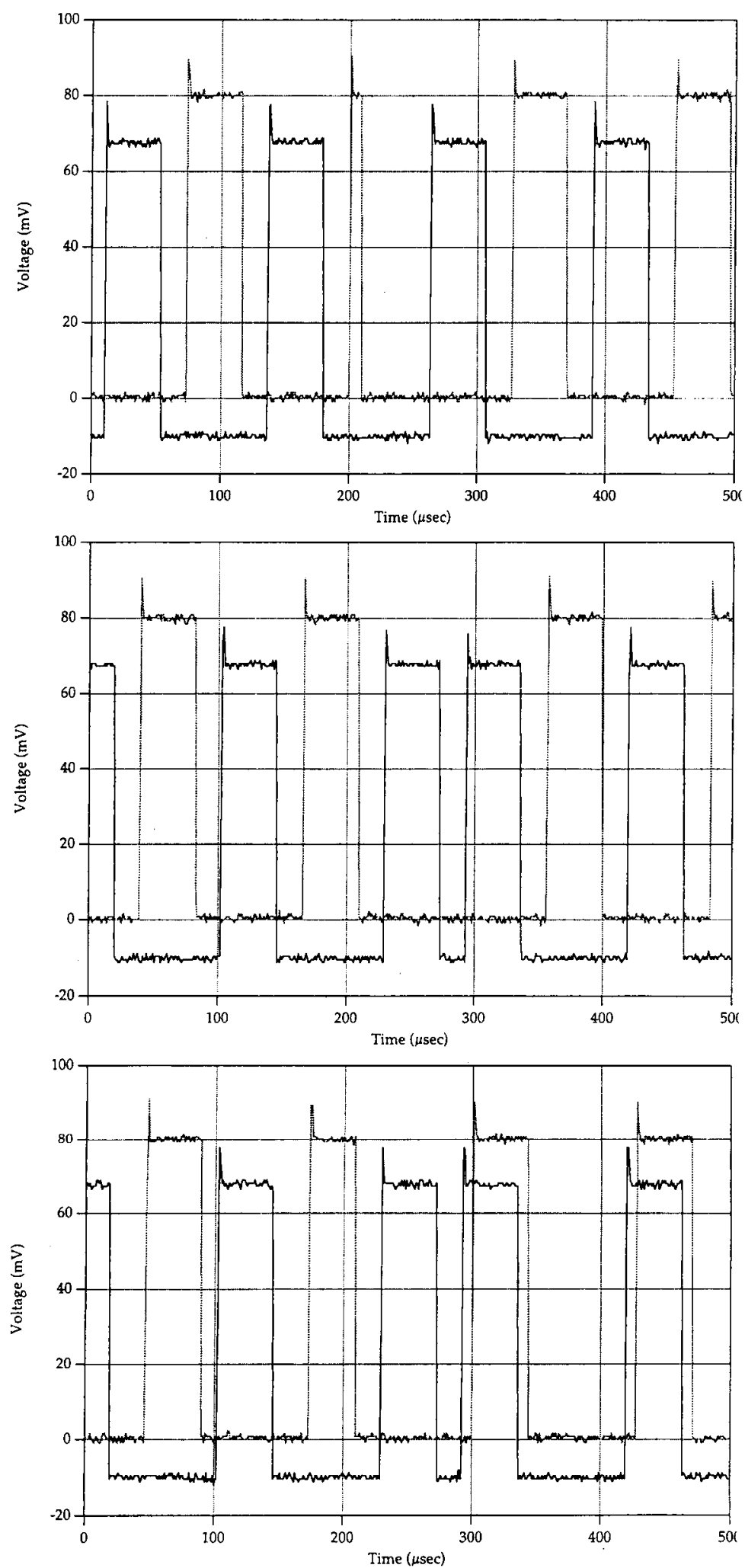

Figure 6. Sample outputs showing simple (top), double (middle), and overlapping (bottom) events. Operation was in Free-run mode and the LET was 8.6. 